\title{
Simple vs complex models for solar cells
}

\author{
Andreea Săbăduș ${ }^{1, *}$, and Marius Paulescu ${ }^{1}$ \\ ${ }^{1}$ Faculty of Physics, West University of Timisoara, V Parvan 4, 300223, Timisoara, Romania
}

\begin{abstract}
In this paper three different procedures for extracting the current-voltage characteristics of a crystalline photovoltaic module are studied. Each procedure is associated to a solar cell model characterized by a well-defined degree of complexity. The results emphasize that the simple models approximate the current-voltage characteristics of a solar cell as good as the more complex models. Even if all the procedures analysed in this paper approximate well the measured characteristics, the specific model parameters experience a large dispersion. From a broader perspective, the results raise a question mark on the ability of the current procedures to accurately extract the solar cell parameters.
\end{abstract}

\section{Introduction}

The photovoltaic (PV) modules are usually delivered by manufacturers accompanied by datasheets. Among other information, three notable points of the currentvoltage (I-V) characteristic are listed on a datasheet: the short-circuit current, the open-circuit voltage and the maximum power point (MPP). All these points are measured in standard test conditions (STC), which are defined by the in-plane solar irradiance of $1000 \mathrm{~W} / \mathrm{m}^{2}$ at normal incidence with the spectral distribution AM1.5G and a solar cell operation temperature of $25^{\circ} \mathrm{C}$. By using such scarce information, the mathematical description of the I-V characteristic of a solar cell is always a challenge [1-3].

In time, different procedures were developed for extracting solar cell parameters. In the last 7 years over 100 of papers were published on the topic of parameters extraction of a solar cell [4].

In this paper three procedures for extracting the I-V characteristics of a crystalline solar cell are studied. Each procedure is associated to a solar cell model characterized by a well-defined degree of complexity. The novelty elements of this report are related to the observations that different sets of parameters lead to an accurate estimation of the I-V characteristic. From this perspective, it is expected that the simpler procedures for extracting the cell parameters to perform better than the more sophisticated ones, which tend many times to local solutions. The rest of the paper is organized as follows. Section 2 presents a brief introduction in the solar cells modeling, emphasizing the models ranking according to the complexity of the I-V characteristic description. Section 3 is devoted to analyzing the results while the main conclusions are gathered in Sec. 4.

\footnotetext{
* Corresponding author: andreea.sabadus94@e-uvt.ro
}

\section{Theory}

The current-voltage characteristic of a PV module represents a superposition of the current-voltage characteristics of its constituent solar cells. Many solar cell models have as starting point the Shockley theory of illuminated p-n junction [5]. As a result, the equivalent circuit of the solar cell may be described at different level of approximation (Fig. 1). The typical approximations are the two-diode model and the onediode model. The two-diode model (Fig. 1a) is the most complex, the I-V characteristics being described by a set of seven parameters: the photocurrent, the serial and the shunt resistances and two sets of parameters for each diode (the saturations current and the diode ideality factor). The one-diode model is widely used representing a trade-off between simplicity and accuracy. This model can be divided into two different categories: the ideal model (Fig. 1c), that neglects the series and shunt resistances and the full one-diode (Fig. 1b).

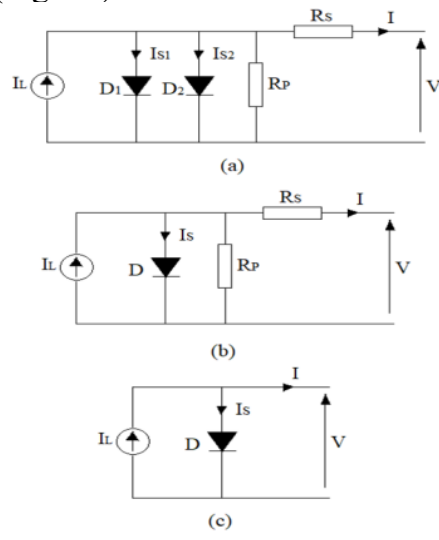

Fig. 1. Equivalent electrical circuit of a solar cell: (a) twodiode model, (b) one-diode model, (c) ideal model. 
The current-voltage characteristics within the twodiode model of a solar cell is given by the equation:

$$
\begin{aligned}
I=I_{L}-I_{S 1}\left[e^{\frac{q\left(V+I R_{S}\right)}{m_{1} k_{B} T}}-1\right]- \\
\quad-I_{S 2}\left[e^{\frac{q\left(V+I R_{S}\right)}{m_{2} k_{B} T}}-1\right]-\frac{V+I R_{S}}{R_{p}}
\end{aligned}
$$

where:

$$
\begin{array}{ll}
\text { - } & I_{L}[A] \text { is the photocurrent generated at STC } \\
\text { - } & I_{S}[A] \text { is the diode saturation current } \\
\text { - } & m \text { is the diode ideality factor } \\
\text { - } & R_{S}[\Omega] \text { is the series resistance } \\
\text { - } & R_{P}[\Omega] \text { is the shunt resistance } \\
\text { - } & q \text { is the electron charge } \\
\text { - } & k_{B} \text { is the Boltzmann constant } \\
& T \text { is the cell temperature }
\end{array}
$$

The current-voltage of a solar within the one-diode model is given by:

$$
I=I_{L}-I_{S}\left[e^{\frac{q\left(V+I R_{S}\right)}{m k_{B} T}}-1\right]-\frac{V+l R_{S}}{R_{P}}
$$

For the ideal model, the current-voltage characteristics reduces to:

$$
I=I_{L}-I_{S}\left[e^{\frac{q V}{m k_{B} T}}-1\right]
$$

Extracting the parameters of the I-V characteristics means to find the appropriate parameters in Eqs. (1-3) for which the modeled and measured characteristics overlap. Solving this problem becomes a very hard task when only the datasheet of PV module is available.

\subsection{One-diode model}

\subsubsection{Ideal model}

The ideal model is the simplest approximation of a solar cell. The number of parameters that describes the $\mathrm{I}-\mathrm{V}$ characteristic is three: the photocurrent, the diode saturation current and the diode ideality factor. The parameters can be calculated directly by solving the system of equation obtained by applying Eq. (3) in the three notable points from datasheet. Since the system of equation is highly non-linear, a numerical procedure for solving the system should be applied. The guess values of the parameters, which are used further to run an algorithm, are estimated taken into account the following assumptions: (1) The photogenerated current and the short-circuit current are roughly equal, (2) $\exp \left(\frac{q V_{O C}}{k_{B} T}\right) \gg 1$ and (3) an ideal p-n junction: $m=1$.

$$
\begin{gathered}
I_{L}=I_{S C} \\
I_{S}=I_{S C^{e}} e^{-\frac{q V_{O C}}{k_{B} T}} \\
m=1
\end{gathered}
$$

\subsubsection{Four-parameter model}

The model contains four parameters $\left(I_{L}, I_{S}, m, R_{S}\right)$. The model derives from the one-diode model, under the simplifying assumption that the shunt resistance is infinite $\left(R_{p}=\infty\right)$. This assumption is realistic, given the small influence of $R_{p}$ on the I-V characteristic of a crystalline solar cell. The model equation is:

$$
I=I_{L}-I_{S}\left[e^{\frac{q\left(V+l R_{S}\right)}{m k_{B} T}}-1\right]
$$

The system of equation for the model parameters is obtained by applying Eq. (5) in the notable points listed in datasheet and taking into account that one of these points is the maximum power point (MPP). Thus, a fourth equation can be written by cancelling the power derivative in MPP. The guess values required for starting the numerical algorithm are:

$$
\begin{gathered}
I_{L}=I_{S C} \\
I_{S}=I_{S C^{e}}{ }^{-\frac{q V_{O C}}{m k_{B} T}} \\
m=1.5 \\
R_{S}=\frac{V_{M}\left(\frac{I_{S C}}{I_{M}}-1\right)+\frac{V_{O C}-V_{M}}{\ln \left(1-\frac{I_{M}}{I_{S C}}\right)}}{I_{S C}-I_{M}+\frac{I_{M}}{\ln \left(1-\frac{I_{M}}{I_{S C}}\right)}}
\end{gathered}
$$

\subsubsection{Five-parameter model}

This model takes into account all the parameters of the one-diode model. This is proper one-diode model. The number of parameters in this case is five. Equation (2) describes the I-V characteristic. Supplementary assumptions are required for writing the system of equations (e.g. [1]). Solving the resulted non-linear system is always a challenge. In this study we used the Newton-Raphson algorithm for function optimization. It is an iterative algorithm aiming to minimize the objective function defined as follows:

$$
S\left(I_{L}, I_{S}, m, R_{S}, R_{p}\right)=\sum_{i=1}^{N}\left(I_{i}^{e x p}-I_{i}^{\text {th }}\right)^{2}
$$

$l_{i}^{\exp }$ is the $i^{\text {th }}$ sample out of $N$ experimental current data sample while $I_{i}^{\text {th }}$ is the $i^{\text {th }}$ sample of the estimated current. The guess values required for starting the algorithm are:

$$
\begin{gathered}
I_{L}=I_{S C} \\
I_{S}=\frac{I_{S C}-\frac{V_{O C}}{R_{P}}}{e^{\frac{q V_{O C}}{m k_{B} T}}-1} \\
m=1.5 \\
R_{S}=-\left.\left(\frac{\partial I}{\partial V}\right)^{-1}\right|_{V=V_{O C}} \\
R_{P}=-\left.\left(\frac{\partial I}{\partial V}\right)^{-1}\right|_{l=I_{S C}}
\end{gathered}
$$




\section{Results}

In this study the three models presented in Section 2 are analysed within the frame of a numerical experiment. The models were implemented in computer codes and run for finding the I-V characteristic of the Kyocera KC200GT PV module. The numerical procedures were conducted on basis of data included in the datasheet of the PV module. The values of the short-circuit current, open-circuit voltage and maximum power point were used as inputs, for generating the system of equations. The solution was compared with the measured I-V characteristic provided by the manufacturer. The fit of the estimated and measured curves was assessed using the determination coefficient $r^{2}$. The determination coefficient is a measure of how well a model fits a set of data:

$$
r^{2}=1-\frac{\sum_{i=1}^{M}\left(m_{i}-c_{i}\right)^{2}}{\sum_{i=1}^{M}\left(m_{i}-\mu\right)^{2}}
$$

where $c$ and $m$ refer to the computed and measured values, respectively, while $M$ is the sample size.

Table 1 shows the results. The numerical algorithms were developed within the mathematical software MathCAD [6] while the I-V characteristic from the datasheet was digitized using GetDataGraph tool [7]. Looking at the results, it can be seen that the coefficient of determination falls in the range 0.9820.995, demonstrating the measured $\mathrm{I}-\mathrm{V}$ curve is approximated very well by any of the three models. This is confirmed visually in Figure 2.

The estimated parameters obtained with the fiveparameter procedure are also compared with the ones previously reported by other studies [8-12]. Ref. [8] use a methodology based on imperialist comparative algorithm. To obtain the five parameters, Ref. [9] applied a multi-verse optimization metaheuristic approach. Ref. [10] considered an optimization algorithm that required the determination of a vector solution, a search range and an objective function. In addition to the three notable points from the currentvoltage characteristic, the method used by Ref. [11] needs the slopes of the current-voltage curve at shortcircuit current and open-circuit voltage to calculate the five parameters. Ref. [12] presents a complex work, using an iterative algorithm to calculate the parameters and a simplification so the solution can be calculated analytically. Also, a very good overlapping of the I-V characteristic can be observed in Fig. 3.

Even if all the procedures approximate well the measured I-V characteristic the parameters experience a large dispersion. The diode saturation current dispersion covers several orders of magnitude. Figure 4 captures this behaviour.
Table 1. The one-diode model parameters and its accuracy in the fitting of the experimental I-V characteristic of the considered PV module. The results are evaluated at STC.

\begin{tabular}{|c|c|c|c|}
\hline Model & Parameters & Value & $r^{2}$ \\
\hline \multirow{5}{*}{$\begin{array}{l}\text { This work } \\
\text { 3-param }\end{array}$} & $I_{L}[A]$ & 8.21 & \multirow{5}{*}{0.995} \\
\hline & $I_{S}[A]$ & $1.781 \cdot 10^{-5}$ & \\
\hline & $m$ & 1.818 & \\
\hline & $R_{S}[\Omega]$ & - & \\
\hline & $R_{p}[\Omega]$ & - & \\
\hline \multirow{5}{*}{$\begin{array}{l}\text { This work } \\
\text { 4-param }\end{array}$} & $I_{L}[A]$ & 8.21 & \multirow{5}{*}{0.993} \\
\hline & $I_{S}[A]$ & $3.414 \cdot 10^{-5}$ & \\
\hline & $m$ & 1.914 & \\
\hline & $R_{S}[\Omega]$ & $6.782 \cdot 10^{-4}$ & \\
\hline & $R_{p}[\Omega]$ & - & \\
\hline \multirow{5}{*}{$\begin{array}{l}\text { This work } \\
\text { 5-param }\end{array}$} & $I_{L}[A]$ & 8.21 & \multirow{5}{*}{0.982} \\
\hline & $I_{S}[A]$ & $9.901 \cdot 10^{-8}$ & \\
\hline & $m$ & 1.300 & \\
\hline & $R_{S}[\Omega]$ & $4.185 \cdot 10^{-3}$ & \\
\hline & $R_{p}[\Omega]$ & 10.147 & \\
\hline
\end{tabular}

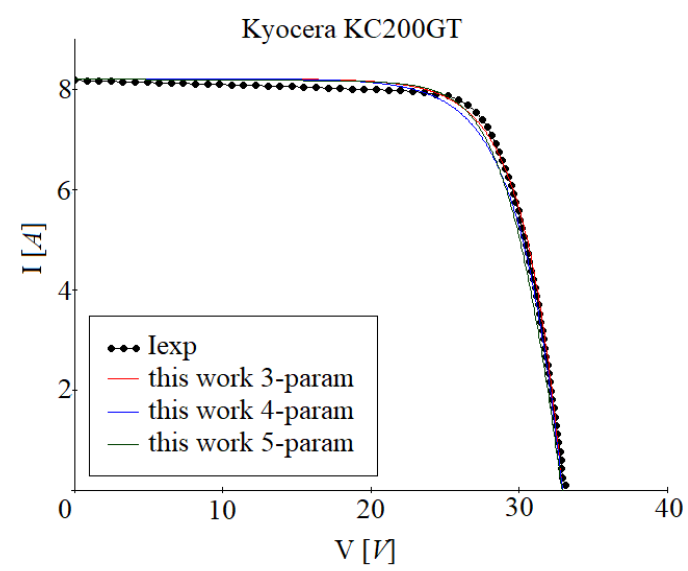

Fig. 2. Measured and estimated I-V characteristic of Kyocera KC200GT PV module.

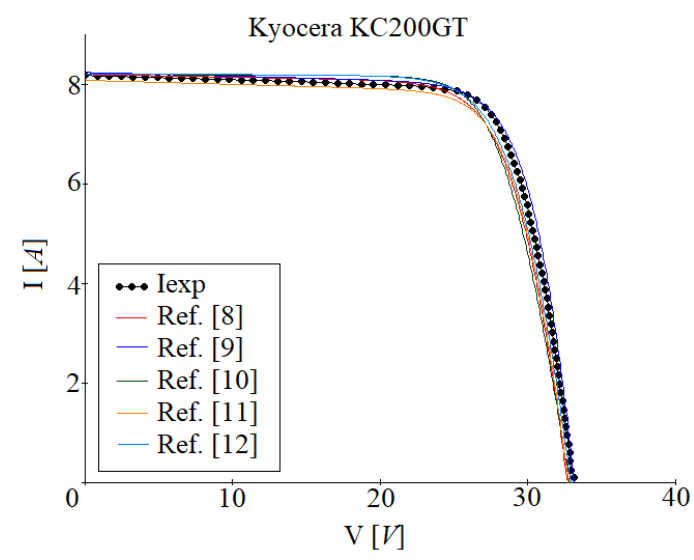

Fig. 3. Measured and estimated I-V characteristic of Kyocera KC200GT PV module using the parameters reported by Ref. [8-12]. 

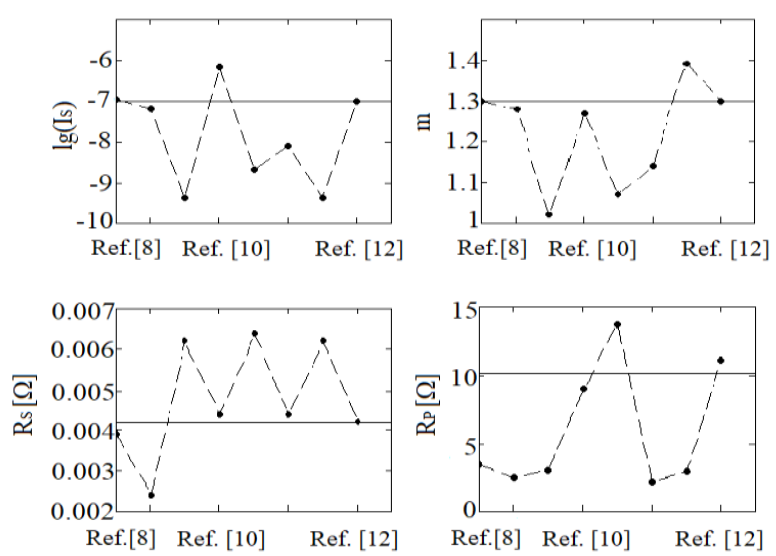

Fig. 4. Variation of the estimated parameters obtained in Ref. [8-12] (dots) and in this work (solid line). The diode saturation current is in logarithmic scale.

From a broader perspective, the results raise a question mark on the ability of the current procedures for I-V characteristics, to extract the solar cell model parameters. This study confirms our previous results [13], namely that, in general, the procedures were developed aiming to model the output power delivered by a PV module to different external loads and does not guarantee the accurate identification of the parameters.

\section{Conclusions}

In this study three simple procedures for extracting the I-V characteristic of a PV module at STC were discussed. The procedures are developed within the frame of the single-diode model. One procedure solves the three-parameter model, one the four-parameter model and other the five-parameter model. The results were assessed against the measured I-V characteristic of a commercially available PV module. The estimated parameters were compared with the ones previously reported. A remarkable conclusion can be taken. Simple models approximate the I-V characteristic of a PV module as good as a more complex model. The main advantages of a simple procedure are: the numerical solution of the high non-linear system of equations converges to a global solution (increasing the confidence in the estimated parameters) and the computer algorithm runs faster.

This work was supported by a grant from 32-ELI/01.09.2016 ELICRYS-2 RO-CERN Programme, ELI-NP Domain, Physical and numerical experiments for studying LPA and their interaction with crystalline materials.

\section{References}

1. V. Lo Brano, A. Orioli, G. Ciulla, A. Di Gangi, Sol Energy Mater Sol Cells 94, 1358-1370 (2010)
2. D.T. Cotfas, P.A. Cotfas, S. Kaplanis, Renew Sustain Energy Rev 28, 588-596 (2010)

3. J. Appelbaum, A. Peled, Sol Energy Mater Sol Cells 122, 164-173 (2014)

4. R. Abbassi, A. Abbassi, M. Jemli, S. Chebbi, Renew Sustain Energy Rev 90, 453-474 (2018)

5. S. Lineykin, M. Averbukh, A. Kuperman, Renew Sustain Energy Rev 30, 282-289 (2014)

6. PTC - MathCAD -Engineering Calculation Software. http://www.ptc.com/products/ mathcad/. Accessed June 2018

7. GetDataGraph - Tool for digitizing graphs and images. http://getdata-graph-digitizer.com. Accessed June 2018

8. A. Fathy, H. Rezk, Renew Energy 111, 307-320 (2017)

9. E.E. Ali, M.A. El-Hameed, A.A. El-Fergany, M.M. El-Arini, Sustain Energy Technol Asses 17, 68-76 (2016)

10. D.F. Alam, D.A. Yousri, M.B. Eteiba, Energy Convers Manage 101, 410-422 (2015)

11. O. Mares, M. Paulescu, V. Badescu, Energy Convers Manage 105, 139-148 (2015)

12. K. Ishaque, Z. Salam, H. Taheri, Sol Energy Mater Sol Cells 95, 586-594 (2011)

13. A. Sabadus, M. Paulescu, V. Badescu. In Proc. of the Conference for Sustainable Energy (CSE) Brasov (I. Vișa, A. Duța Eds.), Nearly zero Energy Community, Springer Proceedings in Energy, pp. 434-442 (2017). 